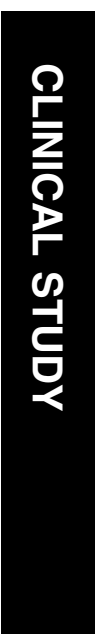

\section{Tacrolimus ointment vs steroid ointment for eyelid dermatitis in patients with atopic keratoconjunctivitis}

\begin{abstract}
Aims The main objective of this explorative study was to evaluate if tacrolimus ointment could be safer than corticosteroid ointment, with special reference to the intraocular pressure in the treatment of eyelid eczema in patients with atopic keratoconjunctivitis
\end{abstract} (AKC). Secondary aims were to compare the effects of the treatments on eyelid eczema and their potential impact on ocular surface inflammation.

Methods Tacrolimus $0.1 \%$ ointment and clobetasone butyrate $0.05 \%$ ointment were compared in a double-masked explorative crossover study. In total, 25 AKC patients were included. Each ointment was applied twice daily for 3 weeks, with 2 weeks of washout before, between, and after treatments. Efficacy was determined by eye examination and the patients' own symptom scoring. Cytology and cytokine measurements were performed on tear samples. Safety parameters were intraocular pressure, presence of bacteria and fungi, and the patients' reports of adverse events. The validity of the crossover design was explored with analysis of variance, and the effect of each medication was calculated with paired $t$-test and Wilcoxon paired test. Results A total of 20 patients completed the study. Both treatments were effective in reducing signs and symptoms of eyelid eczema, with a near superior benefit for tacrolimus in terms of eczema (total skin score) signs $(P=0.05)$. No serious adverse events occurred and interestingly, intraocular pressure was not evidently affected by either treatment.

Conclusion Tacrolimus $0.1 \%$ ointment is a promising alternative therapy for eyelid eczema in AKC patients. Long-term studies are
E Nivenius ${ }^{1}$, I van der Ploeg ${ }^{1}$, K Jung ${ }^{2}$, E Chryssanthou' ${ }^{2}$ M van $\mathrm{Hage}^{3}$ and PG Montan' needed to further determine the value of tacrolimus in this patient group.

Eye (2007) 21, 968-975; doi:10.1038/sj.eye.6702367; published online 5 May 2006

Keywords: atopic keratoconjunctivitis; steroids; cytokine; eyelid eczema; intraocular pressure; tacrolimus

\section{Introduction}

Eyelid eczema as part of the atopic dermatitis syndrome (AD) constitutes a therapeutic challenge. In general, the dermatitis has a chronic course with exacerbations causing considerable discomfort and cosmetic problems. The inflammatory activity of the eyelid margins may interact with the ocular surface-

aggravating conjunctivitis and keratitis, the typical and potentially sight-threatening ocular manifestations of the syndrome, named atopic keratoconjunctivitis (AKC). ${ }^{1}$ There are no available evidence-based therapeutic guidelines for eyelid eczema. Different or even conflicting management approaches may result from the fact that not only ophthalmologists but also dermatologists, allergists, and general practitioners provide care. Apart from emollients, the backbone of treatment for eyelid eczema is steroid ointments. Systemic immunesuppressants may be considered in more severe cases. There is a restriction for use of steroid ointments near the eye, warranted mainly by the propensity of steroid-containing eye-drops to cause increased intraocular pressure (IOP). In the worst-case scenario, this rise in IOP can cause irreversible glaucoma. Approximately $18-36 \%$ of the general population respond to steroid eye drops, with most often moderate elevations even after a short-term 
administration..$^{2-5}$ With steroid application on eyelid skin, increased IOP seems less common, but there are no controlled prospective studies available and several case studies attest to the fact that in long-term treatment, this risk should not be overlooked. ${ }^{6-8}$ As a consequence, regular ophthalmologic examinations are recommended. Another feared complication following prolonged steroid treatment is skin atrophy. ${ }^{9}$ As an alternative to steroids, tacrolimus ointment is a valid therapeutic option for atopic dermatitis since more than 5 years. ${ }^{10-12}$ Successful treatment of eyelid eczema in AKC patients has further been documented in case series ${ }^{7,13}$ and three noncontrolled open-label studies. ${ }^{14-16}$ To the best of our knowledge, no comparative trial of tacrolimus ointment and steroid ointment for eyelid dermatitis has been performed. Moreover, no studies of inflammatory cells and cytokines in tears in response to these treatments have been presented. With the aim of investigating the potential benefits and side effects of tacrolimus in comparison with 'standard' care, that is a mid-potent steroid formulation, a prospective, randomized, double-blind crossover study was undertaken.

\section{Materials and methods}

\section{Subjects}

According to the defined criteria (Table 1), patients with a clinical diagnosis of AKC and who were regularly seen by one of the investigators (PM) in the outpatient clinic of St Erik's Eye Hospital were recruited. Written informed consent was obtained from each participant and the local ethics committee approved the project.

\section{Study design}

Tacrolimus ointment $0.1 \%$ (Protopic ${ }^{\circledR}$, Fujisawa $\mathrm{GmbH}$, Munich, Germany) and a mid-potent steroid clobetasone butyrate $0.05 \%$ (Emovat ${ }^{\circledR}$, GlaxoSmithKline $\mathrm{AB}$ Mölndal, Sweden) were study treatments. Each ointment was given twice daily on the eyelids of both eyes for a 3week period in a double-masked crossover manner to each participant. No other topical, periorbital, or systemic anti-inflammatory or anti-infective treatment was allowed during the study or during the washout periods (Figure 1). The trial was conducted from January to April 2004 and included five visits (Figure 1). At visit 1, after the initial 2-week washout, study eligibility according to inclusion and exclusion criteria (Table 1) was confirmed, and the patients were randomized to receive one of the study drugs. A research nurse managed randomization and drug distribution. Every visit included visual acuity testing, scoring of signs of eyelid dermatitis, blepharitis, conjunctivitis and keratitis, measurement of IOP, tear sampling for cytology and cytokine assessments, sampling for bacteria and fungi, and collections of patient diaries, vide infra. At the first visit, a serum sample was taken to analyse immunoglobulin E (IgE) antibodies against Staphylococcal enterotoxin B (SEB).

\section{Assessment of symptoms and signs}

A score system modified from European Tacrolimus Multicenter Atopic Dermatitis Study Group ${ }^{17}$ was designed for eyelid and eyelid margin signs. As for eyelid eczema, the following parameters were assessed: oedema, redness, excoriations, crusts/oozing, and lichenification. For blepharitis, oedema, redness, and crusts/oozing were assessed. All signs were scored 0-3: 0: no, 1: mild, 2: moderate, and 3: severe signs.

Concerning ocular surface signs, the following grading system was used: Conjunctivitis: 0 , no changes; 1 , mild bulbar injection and superior sub tarsal fibrosis; 2 , moderate mixed bulbar injection; and 3, severe mixed bulbar injection Keratitis: 0, no corneal affection; 1, a few

Table 1 Inclusion and exclusion criteria

\begin{tabular}{ll}
\hline Inclusion criteria & Exclusion criteria \\
\hline AKC with eyelid eczema and need for topical and/or periorbital & $\begin{array}{l}\text { Need for continuous local or systemic anti-inflammatory } \\
\text { steroid treatment during the last } 6 \text { months }\end{array}$ \\
$\begin{array}{l}\text { before the study and } \\
\text { ability to sustain } 2 \text { weeks of washout of }\end{array}$ & One functioning eye only \\
any anti-inflammatory and/or anti-infective treatment & Glaucoma diagnosis or IOP $>25$ mmHg \\
\hline
\end{tabular}

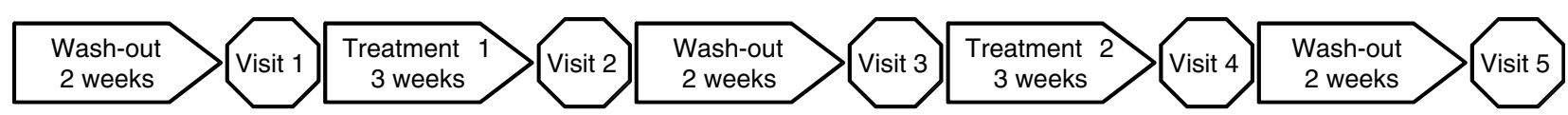

Figure 1 Study design. 
superficial punctuate erosions; 2 , multiple superficial punctuate erosions; and 3, confluent corneal ulcer. All patients had bilateral disease, and scores were recorded from both eyes and added together to obtain as robust data as possible. Maximal score for eyelid eczema was $15+15$, for blepharitis $9+9$, conjunctivitis $3+3$, and for keratitis $3+3$. One observer (EN) performed the examinations and evaluations at all but five of 105 occasions, and a second observer (PM) performed the remaining evaluations. The investigator did not have access to previous measurements for any of the parameters.

In terms of subjective symptoms, the patients were instructed to record eye region discomfort, regardless of type of sensation, twice daily on a visual analogue scale (VAS) graded 0-10: 0 corresponding to no discomfort and 10 to severe discomfort. The VAS scores, adverse events, and treatment application were documented in a diary. VAS scores from the examination days only were used for calculations.

\section{IOP measurements}

To ensure correct measuring of IOP, the same Goldmann tonometer was used at each visit. The calibration was controlled every week with prescribed equipment from Haag-Streit (Bern, Switzerland). To avoid influence of the circadian pressure rhythm, the patients were always examined at a specific time in the morning. The pressure was measured in both eyes and the mean was used for calculations. We defined an increase of $2 \mathrm{mmHg}$ as a clinically relevant increase in IOP, see Statistics section.

\section{Analyses of bacteria and assessment of cytology and cytokines}

The presence of aerobic bacteria on eyelids, eyelid margins, and conjunctiva and the presence of Malassezia species on eyelid and eyelid margins were analysed as previously described. ${ }^{18}$

Tear fluid was collected from the external canthus with a glass blood capillary tube and transferred to Eppendorff tubes. A measure of $2 \mu \mathrm{l}$ were directly fixed with $2 \mu \mathrm{l}$ of $4 \%$ formaldehyde for cytology analysis according to a previously reported method. ${ }^{18}$ One masked observer (EN) counted the eosinophil and neutrophil numbers.

Tear fluid $(30 \mu \mathrm{l})$ was frozen on dry ice and stored at $-80^{\circ} \mathrm{C}$ until analysis for cytokines. The levels of IL- $1 \beta$, IL-2, IL-4, IL-5, IL-6, IL-7, IL-8, IL-10, IL-12 (p70), IL-13, GM-CSF, IFN- $\gamma$, and TNF- $\alpha$ were measured with Human Cytokine Premixed LINCOplex HCYTO-60K-PMX Kit from Linco Research Inc. (St Charles, MO, USA), according to the instructions and read on a Luminex ${ }^{\mathrm{TM}}$
100 instrument from Luminex corporation (Austin, TX, USA). The tear samples were diluted 1:2 with assay buffer and run in duplicates. Out of the 13 cytokines in the kit, IL-2, IL-4, IL-5, IL-8, IL-10, IL-12, IL-13, IFN- $\gamma$, and TNF- $\alpha$ were chosen for further analyses.

Serum IgE against SEB was assessed with the Pharmacia CAP System (Pharmacia Diagnostics AB, Uppsala, Sweden), according to the instructions of the manufacturer. A positive test was defined as $0.35 \mathrm{k}_{\mathrm{A}} \mathrm{U} / \mathrm{L}$.

\section{Statistics and sample size}

The study was designed with the assumption that there would be a $30 \%$ proportion of steroid-treated patients with a clinically relevant rise of IOP, that is $>2 \mathrm{mmHg}$, as opposed to no such rise in the tacrolimus group, leading to a statistically significant difference $(P<0.05)$ with the sign test for a sample of 22 individuals in a crossover study. In view of the crossover design, data from visits 1 , 3 , and 5 were considered as baseline values (Figure 1) and explored with analysis of variance (ANOVA) to exclude significant carryover or rebound phenomena of the treatments (Table 2). All post-treatment values were compared with the baseline values and analysed with parametric and nonparametric tests for dependent variables (paired $t$-test and Wilcoxon paired test) to

Table 2 Validity of the crossover design by control of baseline values

\begin{tabular}{lc}
\hline Parameter & $\mathrm{P}$ \\
\hline IOP & 0.278 \\
Subjective score & 0.278 \\
Total skin score & 0.107 \\
Eczema & 0.157 \\
Blepharitis & 0.375 \\
& \\
Total eye score & 0.022 \\
Conjunctivitis & 0.054 \\
Keratitis & 0.142 \\
& \\
Eosinophils & 0.211 \\
Granulocytes & 0.236 \\
& \\
IL-2 & 0.039 \\
IL-4 & 0.187 \\
IL-5 & 0.171 \\
IL-8 & 0.236 \\
IL-10 & 0.035 \\
IL-13 & 0.062 \\
IFN- $\gamma$ & 0.028 \\
TNF- $\alpha$ & 0.019 \\
\hline$P=$ probability value of differences between the visits 1, 3, and 5 & analysed \\
with ANOVA. & \\
&
\end{tabular}


Table 3 Effect of treatment and differences between treatments

\begin{tabular}{|c|c|c|c|c|c|}
\hline Parameter & Fitted baseline mean (SE) & Treatment & Effect on parameter & $C I$ & $\mathrm{P}$ \\
\hline \multirow[t]{3}{*}{ IOP (mmHg) } & \multirow[t]{3}{*}{$12.38(0.37)$} & $\mathrm{T}$ & 0.08 & $-0.74,0.90$ & 0.849 \\
\hline & & $S$ & 0.05 & $-0.66,0.75$ & 0.899 \\
\hline & & T-S & 0.03 & $-1.05,1.12$ & 0.950 \\
\hline \multirow[t]{3}{*}{ Subjective score } & \multirow[t]{3}{*}{$4.34(0.24)$} & $\mathrm{T}$ & -2.13 & $-2.99,-1.27$ & $<0.001$ \\
\hline & & S & -1.86 & $-2.64,-1.08$ & $<0.001$ \\
\hline & & T-S & -0.27 & $-1.42,0.88$ & 0.640 \\
\hline \multirow[t]{3}{*}{ Total skin score ${ }^{\mathrm{a}}$} & \multirow[t]{3}{*}{$14.05(0.63)$} & $\mathrm{T}$ & -6.87 & $-8.48,-5.26$ & $<0.001$ \\
\hline & & S & -4.48 & $-6.28,-2.67$ & $<0.001$ \\
\hline & & T-S & -2.39 & $-4.79,0.00$ & 0.050 \\
\hline \multirow[t]{3}{*}{ Eczema score } & \multirow[t]{3}{*}{$7.29(0.35)$} & $\mathrm{T}$ & -4.10 & $-5.20,-3.00$ & $<0.001$ \\
\hline & & S & -2.66 & $-3.84,-1.48$ & $<0.001$ \\
\hline & & T-S & -1.44 & $-3.05,0.17$ & 0.087 \\
\hline \multirow[t]{3}{*}{ Blepharitis score } & \multirow[t]{3}{*}{$6.76(0.43)$} & $\mathrm{T}$ & -2.72 & $-3.64,-1.80$ & $<0.001$ \\
\hline & & S & -1.82 & $-2.74,-0.90$ & $<0.001$ \\
\hline & & T-S & -0.90 & $-2.19,0.39$ & 0.180 \\
\hline \multirow[t]{3}{*}{ Total eye score ${ }^{\mathrm{b}}$} & \multirow[t]{3}{*}{$3.58(0.16)$} & $\mathrm{T}$ & -0.20 & -0.670 .27 & 0.416 \\
\hline & & $S$ & 0.05 & $-0.42,0.52$ & 0.835 \\
\hline & & T-S & -0.15 & $-0.82,0.52$ & 0.513 \\
\hline \multirow[t]{3}{*}{ Conjunctivitis score } & \multirow[t]{3}{*}{$2.88(0.11)$} & $\mathrm{T}$ & -0.17 & $-0.54,0.20$ & 0.380 \\
\hline & & S & -0.01 & $-0.38,0.36$ & 0.953 \\
\hline & & T-S & -0.16 & $-0.69,0.37$ & 0.556 \\
\hline \multirow[t]{3}{*}{ Keratitis score } & \multirow[t]{3}{*}{$0.85(0.14)$} & $\mathrm{T}$ & -0.04 & $-0.31,0.23$ & 0.794 \\
\hline & & $\mathrm{S}$ & 0.07 & $-0.19,0.33$ & 0.652 \\
\hline & & T-S & -0.11 & $-0.48,0.26$ & 0.566 \\
\hline \multirow[t]{3}{*}{ Eosinophils (cells / $\mu$ l tear fluid) } & \multirow[t]{3}{*}{$25.36(7.54)$} & $\mathrm{T}$ & -10.78 & $-22.56,1.00$ & 0.073 \\
\hline & & S & -8.80 & $-2.79,20.38$ & 0.135 \\
\hline & & T-S & -1.98 & $-16.50,14.54$ & 0.811 \\
\hline \multirow[t]{3}{*}{ Granulocytes (cells / $\mu$ l tear fluid) } & $366.0(92.18)$ & $\mathrm{T}$ & -64.4 & $-270.8,141.95$ & 0.535 \\
\hline & & S & -101.3 & $-319.7,117.1$ & 0.358 \\
\hline & & T-S & -36.91 & $-337.4,263.6$ & 0.806 \\
\hline IL-2 (pg/ml) & $26.94(2.76)$ & $\mathrm{T}$ & 2.05 & $-6.87,10.97$ & 0.648 \\
\hline & & $\mathrm{S}$ & -0.70 & $-8.20,6.80$ & 0.853 \\
\hline & & T-S & 2.75 & $-8.91,14.41$ & 0.638 \\
\hline IL-4 (pg/ml) & $46.02(6.59)$ & $\mathrm{T}$ & 19.52 & $-1.84,40.88$ & 0.074 \\
\hline & & $S$ & 18.37 & $-4.25,41.00$ & 0.111 \\
\hline & & T-S & 1.15 & $-14.41,16.71$ & 0.944 \\
\hline IL-5 (pg/ml) & $1.49(0.50)$ & $\mathrm{T}$ & -1.14 & $-3.82,1.54$ & 0.398 \\
\hline & & $\mathrm{S}$ & -0.53 & $-3.07,2.01$ & 0.679 \\
\hline & & T-S & -0.61 & $-4.31,3.09$ & 0.742 \\
\hline IL-8 (pg/ml) & $367.8(80.65)$ & $\mathrm{T}$ & -263.6 & $-538.6,11.40$ & 0.062 \\
\hline & & $\mathrm{S}$ & -45.92 & $-322.5,230.7$ & 0.741 \\
\hline & & T-S & -190.7 & $-580.7,199.3$ & 0.331 \\
\hline IL-10 (pg/ml) & $55.16(5.54)$ & $\mathrm{T}$ & 3.57 & $-12.05,19.19$ & 0.650 \\
\hline & & S & 4.86 & $-10.32,20.04$ & 0.526 \\
\hline & & T-S & -1.29 & $-23.07,20.49$ & 0.906 \\
\hline IL-13 (pg/ml) & $160.3(18.96)$ & $\mathrm{T}$ & -26.90 & $-77.46,23.66$ & 0.293 \\
\hline & & $S$ & -19.52 & $-77.40,38.36$ & 0.503 \\
\hline & & T-S & -7.38 & $-84.24,69.48$ & 0.848 \\
\hline $\mathrm{IFN}-\gamma(\mathrm{pg} / \mathrm{ml})$ & 106.5 (10.08) & $\mathrm{T}$ & 2.25 & $-23.19,27.69$ & 0.860 \\
\hline & & S & -6.49 & $-27.77,14.79$ & 0.545 \\
\hline & & T-S & 8.74 & $-24.42,41.90$ & 0.599 \\
\hline TNF- $\alpha(\mathrm{pg} / \mathrm{ml})$ & $17.76(1.46)$ & $\mathrm{T}$ & -1.24 & $-4.92,2.44$ & 0.504 \\
\hline & & S & -1.16 & $-4.46,2.14$ & 0.483 \\
\hline & & T-S & -0.08 & $-5.02,4.86$ & 0.513 \\
\hline
\end{tabular}

The effects of tacrolimus treatment (T) steroid treatments (S) and the difference of T-S. Model-based fitted values after elimination of individual effects. $\mathrm{SE}=$ standard error, $\mathrm{CI}=95 \%$ confidence interval, $P=$ probability value.

${ }^{a}$ Total skin score $=$ eyelid eczema + blepharitis score.

${ }^{\mathrm{b}}$ Total eye score $=$ conjunctivitis + keratitis score 
compare the respective treatment effects within each individual (Table 3). Categorical data were analysed with the sign test. Post hoc analyses of baseline global objective scores in relation to treatment preference and to the presence of SEB were performed with the MannWhitney test. Spearman correlation test was used to explore various associations. A $P$-value $<0.05$ was defined as statistically significant.

\section{Results}

Patients, participant flow, assessment of baseline values

Overall, the disease activity in the study group was moderate. Twenty-five patients were recruited, of whom three patients could not withstand the first washout period without using steroid eye drops, and were consequently excluded. The remaining 22 were included and provided with study medication. One patient was lost to follow-up early in the study and another patient was excluded owing to use of steroid ointment as an add-on to study medications. Thus, 20 patients completed the trial (nine females and 11 males; average age \pm SD: $46 \pm 12$ years, range $18-70$ years). Compliance and documentation was generally satisfactory. One participant made a minor protocol deviation by taking oral antibiotics. He was therefore excluded from the microcolonization analyses. Additionally, four subjects took antihistamine tablets on few occasions because of general allergic symptoms. No one discontinued the trial owing to adverse events, but several patients found the washout periods almost intolerable, and two patients had such severe disease that the last period of washout could not be justified. To ascertain the validity of the crossover design, the three baseline values were compared. Neither scores for the eczema or blepharitis nor IOP values, bacterial or fungal flora differed significantly between nontreatment examination days. Conversely, there were significant alterations for ocular signs and for some inflammatory markers in tear fluid between baseline measurements (Table 2).

\section{Effect on signs and symptoms}

Both ointments provided significant improvement for eyelid eczema and blepharitis scores as well as for subjective symptoms (Table 3). No difference in effect between tacrolimus and steroid ointment could be proven, but the total skin (eczema + blepharitis) score was almost statistically significant in favour of tacrolimus, $P=0.05$ (Table 3 ). No significant effect was shown for ocular surface scores after either treatment. Baseline values differed significantly for this parameter (Table 2), and therefore the first treatment period only was further analysed. Once again, no treatment effect was detected, making carryover or rebound effects a less likely explanation of the significant baseline variability. After the last washout, the patients were asked to make a global evaluation of the therapies: four expressed no preference; 11 patients preferred tacrolimus ointment; and five favoured steroid ointment, $P=0.2$. Patients who preferred tacrolimus ointment had higher total objective scores at inclusion, $P=0.03$.

\section{Effect on tear markers of inflammation}

Owing to sampling difficulties and technical failure, $6 / 100$ cytology slides were not amenable to analysis. No effect on eosinophil or neutrophil numbers was proven after either therapy. Four patients were excluded from the cytokine analysis because of insufficient tear volumes. No baseline variability was seen for IL-4, IL-5, IL-8, and IL-13 (Table 2). Trends of reduction for IL-4, $P=0.07$, and IL- $8, P=0.06$ were observed after tacrolimus treatment only (Table 3 ). For the other cytokines, there was a significant variability in analogy with the conjunctivitis scores. A post hoc analysis of the first treatment period revealed that only IL- 2 and TNF- $\alpha$ were significantly responsive to treatment and only to tacrolimus. Consequently, no significant carryover or rebound effects are suspected for the cytokines in general. Expected associations between eosinophil numbers and IL- 5 as well as neutrophil numbers and IL-8 were confirmed (data not shown).

\section{Side effects/tolerance}

IOP was not significantly changed by any of the ointments and no significant difference between the therapies was observed (Table 3 ). For these calculations, the mean pressure between the eyes was used. The IOP response variable, defined as an increase above $2 \mathrm{mmHg}$ in either eye, was noted for $3 / 20$ steroid- and for $5 / 20$ tacrolimus-treated patients, of whom one responded to both treatments. No patient had an asymmetric response, that is a decrease of IOP in one eye and an increase in the other, but some patients had a larger difference in one eye than in the other. Two participants showed an increase of $5 \mathrm{mmHg}$, one on steroid and one on tacrolimus therapy. The pressure was normalized in both cases after washout. Seven patients reported initial skin burning during tacrolimus application, whereas no one on steroid experienced this untoward effect, $P=0.02$. For six of these seven patients, the burning lasted for 2-5 days. In addition, two patients complained about a dry feeling of the eye and eyelids and another patient described fluctuating symptoms with red eyes and tearing during tacrolimus treatment. During steroid 
treatment, eight patients reported increased itching. No other adverse events were reported.

\section{Effects on microcolonization}

Microcolonization was not influenced in a significant way by either treatment. The analysis was qualitative; hence, the presence of different species only and not the actual amount of bacteria could be assessed. Coagulasenegative staphylococci (CONS) were isolated from all patients and Staphylococcus aureus (SA) in 18/19 patients. Also in patients with an almost total clearing of signs and subjective symptoms, both CONS and SA were present. Other bacteria seen in few samples were Haemophilus influenzae, Moraxella catarrhalis, Corynebacterium species, and Propionibacterium acnes. In only two patients, Malassezia sympodialis was isolated. One patient reported a relapse of herpes on the eyelid during washout after tacrolimus treatment. This could not be confirmed as it had resolved before the following visit.

\section{Serum IgE antibodies against S. aureus enterotoxin B antigen}

Five out of 20 patients were tested positive for SEB, median $2.5 \mathrm{k}_{\mathrm{A}} \mathrm{U} / \mathrm{L}$ and range $0.4-17 \mathrm{k}_{\mathrm{A}} \mathrm{U} / \mathrm{L}$. The five patients with positive SEB test had significantly higher disease scores, regarding both eczema and eye surface parameters, than those tested negative $P=0.03$.

\section{Discussion}

There are no evidence-based guidelines for the treatment of eyelid eczema. This explorative short-term comparative crossover study investigated tacrolimus $0.1 \%$ vs a midpotent steroid ointment for eyelid eczema of moderate severity in AKC patients. The main objective was to evaluate whether tacrolimus would be a safer alternative to steroid treatment in the eyelid region by not inducing a rise in IOP. Tacrolimus $0.1 \%$ was chosen because of significantly better effect than that of tacrolimus $0.03 \%$ without increase in adverse events, ${ }^{11,12}$ and because of proven similar anti-inflammatory properties to a midpotent steroid. ${ }^{19}$ The clobetasone butyrate ointment was selected because of the ointment-base with White Vaseline that it shares with the tacrolimus formulation on sale in Sweden, thereby facilitating masking. The 3-week treatment periods were mainly warranted from a safety point-of-view, as we hypothesized that the steroid ointment could induce IOP elevations. Moreover, it is known from previous eczema studies that the onset of the anti-inflammatory effect occurs within the first 2 weeks with both treatments. ${ }^{17,19}$ The 2-week washout was justified from a patient comfort perspective.
Both treatments significantly reduced eczema signs and symptoms in agreement with the literature. Although tacrolimus was associated with tolerance problems, that is transient skin burning, the treatment was preferred by a majority of the patients. The hypothesized increase in IOP of at least $2 \mathrm{mmHg}$ in $30 \%$ of the study population after steroid application could not be confirmed. Interestingly, one subject treated with tacrolimus and one receiving clobetasone butyrate reacted with a marked increase in IOP. The IOP was normalized on check-up, but clearly, this study suggests that the risk for IOP being affected by eyelid immunemodulating ointments needs to be elucidated in longterm settings also for tacrolimus, although previous reports do not indicate such a risk. ${ }^{7,20}$

Results from studies with tacrolimus and steroid have indicated a reduction of SA colonization paralleling a decrease of inflammatory signs, ${ }^{21}$ which could not be confirmed in the present study. The role of SA in AKC is not fully known and there seems to be no simple relation between SA colonization and severity of AKC. ${ }^{18}$ Another aspect of the interaction between bacteria and atopic eczema is the risk for superinfection during immunesuppressive therapy. This was not observed in the present investigation, which is in line with previous findings. ${ }^{22}$ Finally, SEB has been implicated as a disease factor in AD and perhaps also in AKC. ${ }^{18,23}$ Such involvement was again apparent in this study where the subjects who had detectable levels of SEB in the serum had significantly higher disease activity scores than the other subjects.

In patients with $\mathrm{AD}^{24}$ and $\mathrm{AKC}_{,}{ }^{25}$ recurrent herpes simplex infections are not uncommon and a suspected increase of herpes simplex lesions during tacrolimus therapy has been a concern. ${ }^{26,27}$ This complication was therefore particularly examined, but apart from a reported recurrence of herpes on the eyelids, no viral lesions were observed in our trial.

Corneal disease is the major sight-threatening complication of AKC. There are at present no data suggesting that conjunctivitis and keratitis are generated by the eczema or closely interrelated, although in the present study a good correlation was seen between skin and eye affection (data not presented). It is quite plausible that at least blepharitis may induce and promote ocular surface changes in AKC, as has been substantiated in observational studies of other blepharitis entities. ${ }^{28,29}$ A positive effect of extended eyelid treatment with tacrolimus has also been reported on conjunctivitis and keratitis. ${ }^{13,16,20}$ Hence, we wanted to know whether a reduction of ocular surface inflammation could be produced by regional eczema treatment. Moreover, there seems to be a positive relationship between conjunctival disease and inflammatory cells as well as cytokines in 
tear fluid, ${ }^{18}$ which was the reason why these objective measures were included in our protocol. However, neither the clinical signs nor the tear markers were significantly influenced by the study medications. Still, it cannot be entirely ruled out that eyelid eczema ointments may have these 'spin-off' benefits. First and mainly, there may have been too short a treatment period to achieve ocular surface effects. Second, the selection of patients with moderate conjunctivitis and keratitis manifestations and also the number of patients may have been decisive. Third, the cytokine analysis used herein appears to have been less sensitive than the one previously employed by our group. ${ }^{18}$ Finally, a significant effect on tear markers of inflammation could probably not be expected considering the lack of impact on the conjunctiva of the treatments.

In conclusion, both treatment concepts proved efficacious in reducing eczema signs and symptoms while not raising any immediate safety concerns. The lack of proven benefits on the ocular surface disease warrants studies over a longer period of time, preferably including AKC patients with severe manifestations, whose need to administer add-on steroid or cyclosporine eye-drops could be used as an efficacy criterion. Long-term studies are also called for to confirm safety regarding IOP changes.

The effects and safety profile of tacrolimus ointment used in an extended period of time are not known, which explains its current second-line status in AD. A serious safety signal of malignant tumour appearance has emerged for systemic tacrolimus treatment, ${ }^{30}$ but concerning topical administration, several studies have demonstrated only a minimal absorption of the drug. ${ }^{12,17,19}$ However, carcinogenesis at the application site has been shown in a mouse model. ${ }^{31}$ In addition, single incidents of malignancy in humans after topical tacrolimus application have been reported ${ }^{32}$ but to date there is no evidence of an increased risk. ${ }^{33} \mathrm{~A}$ relation between these tumours and UV-light damage is suspected and ultraviolet exposure should hence be avoided during tacrolimus treatment. Until further knowledge is reached, the US Food and Drug administration, FDA, has recommended a 'black box' warning ${ }^{34}$ whereas The European Agency for Evaluation of Medical Products, EMEA, has taken no such action. ${ }^{35}$

Based on the presently reported data, we conclude that tacrolimus ointment is a promising alternative to steroid ointment for eyelid eczema in AKC patients, but the said safety and efficacy aspects warrant further exploration.

\section{Acknowledgements}

We thank Professor K Nordlind, dermatologist at Karolinska University Hospital for accepting the task to be the monitor of the study, research nurse Mikaela
Taube for assistance and help with the double-masked design, Berit Spångberg and Margareta Oscarsson for valuable technical support, Rodica Lenkei for help and advise concerning cytokine analysis, and Bo Nilsson for important statistical help. Grants from Crown Princess Margareta's Foundation for the Visually Impaired (KMA), Stiftelsen Synfrämjandets Forskningsfond, the Swedish Research Council, the Swedish Asthma and Allergy Association, the Swedish Cancer and Allergy Fund, the Hesselman Foundation, the Mieczislaw Hubaczs Foundation for Eye Research and from the Karolinska Institute supported this work. No sponsor was involved in the study.

\section{References}

1 Hogan MJ. Atopic keratoconjunctivitis. Trans Am Acad Ophthalmol Otolaryngol 1952; 50: 265-281.

2 Armaly MF. Effect of corticosteroids on intraocular pressure and fluid dynamics. I. The effect of dexamethasone on the normal eye. Arch Ophthalmol 1963; 70: 482-491.

3 Armaly MF. Effect of corticosteroids on intraocular pressure and fluid dynamics. II. The effect of dexamethasone on the glaucomatous eye. Arch Ophthalmol 1963; 70: 492-499.

4 Becker B. Intraocular pressure response to topical corticosteroids. Invest Ophthalmol 1965; 26: 198-205.

5 Tripathi RC, Parapuram SK, Tripathi BJ, Zhong Y, Chalam KV. Corticosteroids and glaucoma risk. Drugs Aging 1999; 15: 439-450.

6 Aggarwal RK, Potamitis T, Chong NH, Guarro M, Shah P, Kheterpal S. Extensive visual loss with topical facial steroids. Eye 1993; 7: 664-666.

7 Kymionis GD, Tsilimbaris MK, Iliaki OE, Christodoulakis E, Siganos CS, Pallikaris IG. Treatment of atopic eyelid disease using topical tacrolimus following corticosteroid discontinuation in a patient with open-angle glaucoma. Cornea 2004; 23: 828-830.

8 Garrott HM, Walland MJ. Glaucoma from topical corticosteroids to the eyelids. Clin Experiment Ophthalmol 2004; 32: 224-226.

9 Kolbe L, Klingman AM, Schreiner V, Stoudemayer T. Corticosteroid-induced atrophy and barrier impairment measured by non-invasive methods in human skin. Skin Res Technol 2001; 7: 73-77.

10 Fleischer Jr AB, Ling M, Eichenfield L, Satoi Y, Jaracz E, Rico MJ et al. Safety and efficacy of 1 year of tacrolimus ointment monotherapy in adults with atopic dermatitis. The European Tacrolimus Ointment Study Group. Arch Dermatol 2000; 136: 999-1006.

11 Hanifin JM, Ling MR, Langley R, Breneman D, Rafal E. Tacrolimus ointment for the treatment of atopic dermatitis in adult patients: Part I. efficacy. J Am Acad Dermatol 2001; 44: S28-S38.

12 Soter NA, Fleischer Jr AB, Webster GF, Monroe E, Lawrence I. Tacrolimus ointment for the treatment of atopic dermatitis in adult patients: Part II. Safety. J Am Acad Dermatol 2001; 44: S39-S46.

13 Rikkers SM, Holland GN, Drayton GE, Michel FK, Torres MF, Takahashi S. Topical tacrolimus treatment of atopic eyelid disease. Am J Ophthalmol 2003; 135: 297-302. 
14 Freeman AK, Serle J, VanVeldhuisen P, Lind L, Clarke J, Singer $\mathrm{G}$ et al. Tacrolimus ointment in the treatment of eyelid dermatitis. Cutis 2004; 73: 267-271.

15 Kawakita T, Takano Y, Asano-Kato N, Tanaka M, Dogru M, Goto E et al. Quantitative evaluation of eyelid elasticity using the cutometer SEM575 and its clinical application in assessing the efficacy of tacrolimus ointment treatment in eyelid atopic dermatitis. Cornea 2004; 23: 468-471.

16 Mayer K, Reinhard T, Reis A, Bohringer D, Sundmacher R. FK 506 ointment $0.1 \%$ - a new therapeutic option for atopic blepharitis. Clinical trial with 14 patients. Klin Monatsbl Augenheilkd 2001; 218: 733-736.

17 Ruzicka T, Bieber T, Schopf E, Rubins A, Dobozy A, Bos JD et al. A short-term trial of tacrolimus ointment for atopic dermatitis. European Tacrolimus Multicenter Atopic Dermatitis Study Group. N Engl J Med 1997; 18: 816-821.

18 Nivenius E, Montan PG, Chryssanthou E, Jung K, van Hage-Hamsten M, van der Ploeg I. No apparent association between periocular and ocular microcolonization and the degree of inflammation in patients with atopic keratoconjunctivitis. Clin Exp Allergy 2004; 34: 725-730.

19 Reitamo S, Rustin M, Ruzicka T, Cambazard F, Kalimo K, Friedmann PS et al, European Tacrolimus Ointment Study Group. Efficacy and safety of tacrolimus ointment compared with that of hydrocortisone butyrate ointment in adult patients with atopic dermatitis. J Allergy Clin Immunol 2002; 109: 547-555.

20 Reinhard T, Reis A, Mayweg S, Oberhuber H, Mathis G, Sundmacher R. Topical Fk506 in inflammatory corneal and conjunctival diseases. A pilot study. Klin Monatsbl Augenheilkd 2002; 219: 125-131.

21 Remitz A, Kyllonen H, Granlund H, Reitamo S. Tacrolimus ointment reduces staphylococcal colonization of atopic dermatitis lesions. J Allergy Clin Immunol 2001; 107: 196-197.

22 Fleischer Jr AB, Ling M, Eichenfield L, Satoi Y, Jaracz E, Rico MJ et al, Tacrolimus Ointment Study Group. Tacrolimus ointment for the treatment of atopic dermatitis is not associated with an increase in cutaneous infections. $J \mathrm{Am}$ Acad Dermatol 2002; 47: 562-570.

23 Shoji J, Kato H, Kitazawa M, Inada N, Sawa M. Evaluation of staphylococcal enterotoxin-specific IgE antibody in tears in allergic keratoconjunctival disorders. Jpn J Ophthalmol 2003; 47: 609-611.

24 Rystedt I, Strannegard IL, Strannegard O. Recurrent viral infections in patients with past or present atopic dermatitis. Br J Dermatol 1986; 114: 575-582.

25 Garrity JA, Liesegang TJ. complications of atopic dermatitis. Can J Ophthalmol 1984; 19: 21-24.

26 Lubbe J, Saurat JH. Cutaneous infections with herpes simplex virus and tacrolimus ointment. J Am Acad Dermatol 2003; 49: 965-966.

27 Joseph MA, Kaufman HE, Insler M. Topical tacrolimus ointment for treatment of refractory anterior segment inflammatory disorders. Cornea 2005; 24: 417-420.

28 McCulley J, Sciallis GF. Meibomian keratoconjunctivitis. Am J Ophthlmol 1978; 84: 788-792.

29 Culbertson W, Huang AJ, Mandelbaum SH, Pflugfelder SC, Boozalis GT, Miller D. Effective treatment of phlyctenular keratoconjunctivitis with oral tetracycline. Ophthalmology 1993; 100: 1358-1366.

30 Jonas S, Rayes N, Neumann U, Neuhaus R, Bechstein WO, Guckelberger $\mathrm{O}$ et al. De novo malignancies after liver transplantation using tacrolimus-based protocols or cyclosporine-based quadruple immunosuppression with an interleukin-2 receptor antibody or antithymocyte globulin. Cancer 1997; 15: 1141-1150.

31 Niwa Y, Terashima T, Sumi H. Topical application of the immunosuppressant tacrolimus accelerates carcinogenesis in mouse skin. Br J Dermatol 2003; 149: 960-967.

32 Becker JC, Hoube R, Vetter CS, Broecker EB. The carcinogenic potential of tacrolimus ointment beyond immune suppression: a hypothesis creating case report. BMC Cancer 2006; 6(1): 7.

33 Naylor M, Elmets C, Jaracz E, Rico JM. Non-melanoma skin cancer in patients with atopic dermatitis treated with topical tacrolimus. J Dermatol Treat 2005; 16: 149-153.

34 Aaronson DW. The 'black box' warning and allergy drugs. J Allergy Clin Immunol 2006; 117: 40-44.

35 Ormerod AD. Topical tacrolimus and pimecrolimus and the risk of cancer: how much cause for concern? Br J Dermatol 2005; 153: 701-705. 